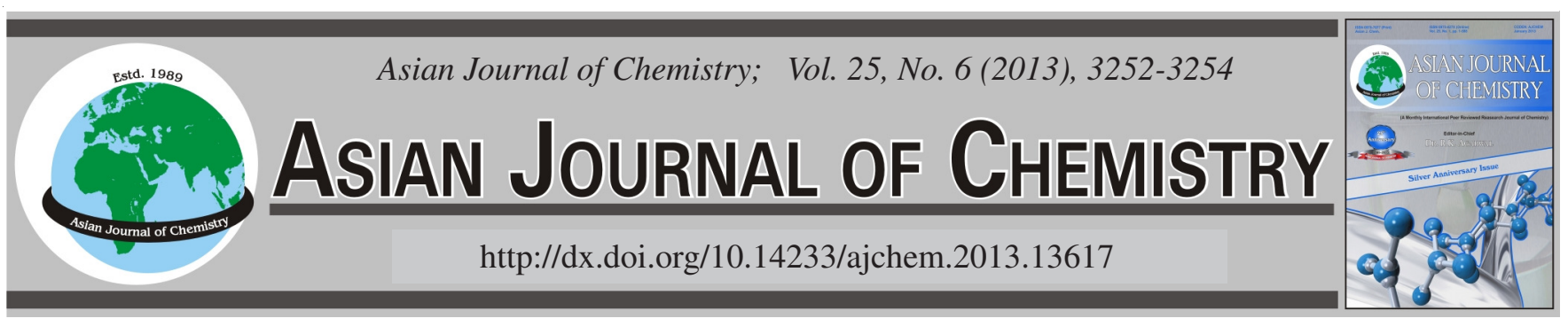

\title{
Analysis of Volatile Oil of Fritillaria cirrhosa D. Don by GC-MS
}

\author{
XIAOJING $\mathrm{WANG}^{1}$ and YUMEI $\mathrm{Li}^{2, *}$
}

${ }^{1}$ School of Chemical Engineering and Technology, Tianjin University, Tianjin 300072, P.R. China

${ }^{2}$ Department of Chemistry, Dezhou University, Shandong 253023, P.R. China

*Corresponding author: E-mail: 2003liyumei@sina.com

(Received: 17 February 2012;

Accepted: 17 December 2012)

AJC-12559

The volatile oil was extracted from Fritillaria cirrhosa D. Don by water steam distillation and the chemical constituents of volatile oil separated were identified by gas chromatography-mass spectrometry analysis. The relative content of the constituents from the volatile oil were determined by area normalization method. Thirty-nine peaks were separated, among which 14 compounds were identified, amounting to $81.92 \%$ of the total amount of volatile oil. The main compounds identified to be 1-octadecene (16.38 \%), 1-dodecene (15.09), oxirane, hexadecyl-(11.38 \%), 1-hexadecanol (10.65\%), 1-eicosanol (7.95\%), 9-octadecynoic acid, methyl ester (6.94 \%), $n$-hexadecanoic acid $(5.41 \%)$. The work provides information for making full use of the Fritillaria cirrhosa D. Don resources and pharmacological activities research of traditional Chinese medicine.

Key Words: Fritillaria cirrhosa D. Don, Volatile oil, Water steam distillation, Gas chromatography-mass spectrometry.

\section{INTRODUCTION}

Fritillaria cirrhosa D. Don is the dried bulb of Fritillaria cirrhosa D.Don, Fritillaria unibracteata Hsiao et K.C. Hsia, Fritillaria przewalskii Maxim., Fritillaria delavayi Franch., which belongs to Liliaceae family ${ }^{1}$ and has been recorded in China pharmacopoeia. It has been used to relieve coughs and eliminate phlegm, etc. The chemical constituents in Fritillaria have been extensively investigated, which contained alkaloids, saponin, terpenoids, steroids, succinic acid, thymidine, adenisine, etc. ${ }^{2-5}$. The chemical and pharmacological research in recent years showed isosteroidal alkaloids are the main bioactive ingredients of Fritillaria species, among which peimine (verticine) and peiminine (verticinone) are the major active alkaloids of Fritillaria cirrhosa D.Don ${ }^{5-9}$ (Fig. 1).

Volatile oil is an important part of medicinal plants for pharmaceutical industries. The further study on chemical components of volatile oil in Fritillaria cirrhosa D.Don has important significance to efficient utilization of Fritillaria cirrhosa D.Don resources. Nevertheless, no reports on volatile oil composition from Fritillaria cirrhosa D.Don have been published. The aim of present work was to investigate the composition of volatile oil by GC-MS from Fritillaria cirrhosa D.Don in order to make full use of the Fritillaria cirrhosa D.Don resources and provide more information for pharmacological activities research of traditional Chinese medicine.

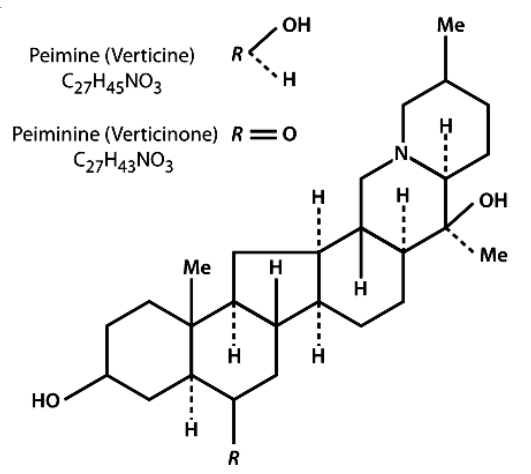

Fig. 1. Chemical structure of peimine and peiminine

\section{EXPERIMENTAL}

PolarisQ GC-MS (thermo-Finnigan): All analytical grade solvents were from Tianjin Fuyu Fine Chemicals Co. Ltd., (Tianjin, China). Anhydrous sodium sulfate was purchased from Beijing Tongguang Fine Chemicals Company, (Beijing, China). Fritillaria cirrhosa D.Don purchased from a local drugstore was ground with a commercial mill to obtain a relatively homogenous drug powder. To determine oil yield of the materials used in the extractions, the powder was dried at $55^{\circ} \mathrm{C}$ until constant weight.

Extraction procedure: Fritillaria cirrhosa D.Don was ground and steam-distilled according to the method recommended by the China Pharmacopoeia. A 100 g Fritillaria 
cirrhosa D.Don was submerged in $500 \mathrm{~mL}$ distilled water for $24 \mathrm{~h}$ and distilled for $10 \mathrm{~h}$. The time of distillation was measured from the beginning of vapour condensation on the walls of condenser. We checked in preliminary experiments that the amount of essential oil collected in separator did not further increase after $8 \mathrm{~h}$ of distillation. Distillate was transferred to separating funnel and was extracted with diethyl ether for 3 times. Extract liquid was collected and reagent was recovered by rotary evaporator. The essential oils were dried over anhydrous sodium sulfate and stored at $4{ }^{\circ} \mathrm{C}$ in the refrigerator. Extractions were performed at least three times and the mean value of the oil yield from Fritillaria cirrhosa D.Don was $1.3 \%$ based on the dried weight.

Analysis of the volatile oils: The volatile oil extracts were analyzed by using gas chromatography-mass spectrometry. The GC-MS conditions were optimized on the basis of the property of the essential oil extracts. Gas Chromatographymass spectrometry was used for identification of volatile compounds in the volatile oil, equipped with a capillary column ZB $5 \mathrm{MS}$ (length $30 \mathrm{~m} \times$ I.D. $0.25 \mathrm{~mm} \times$ thickness $0.25 \mu \mathrm{m}$ ), injector temperature $230{ }^{\circ} \mathrm{C}$. The oven temperature was held at $50{ }^{\circ} \mathrm{C}$ for $3 \mathrm{~min}$ then programmed at $10{ }^{\circ} \mathrm{C} / \mathrm{min}$ to $220{ }^{\circ} \mathrm{C}$ (held for $3 \mathrm{~min}$ ). Helium was used as a carrier gas flowed at a rate of $1 \mathrm{~mL} / \mathrm{min}$. A $1 \mu \mathrm{L}$ of the sample extract was injected using the discharge ratio. Discharge ratio was set at 1:50. The first GC column was used for obtaining the residence time for identification, while the second column and MS detection was used the confirmation. Mass spectra were recorded in EI-source scanning mode with a mass range of 60-400 AMU. Ion source temperature was set at $210^{\circ} \mathrm{C}$ and ionization voltage $70 \mathrm{eV}$.

The identification of the volatile oil components was based on the comparison of their residence time. The percentage composition of the oils was computed by the normalization method from the GC peak areas. The average peak areas of all GC signals were added together and the percentage of each component peak was calculated by comparing its average area to the total areas.

\section{RESULTS AND DISCUSSION}

The sample extracts of Fritillaria cirrhosa D. Don was analyzed by using GC-MS analyses and total ion current chromatograms of volatile oil from Fritillaria cirrhosa D. Don was obtained (Fig. 2). The constituents of the volatile oil from Fritillaria cirrhosa D.Don were identified by comparing their residence time. The relative content of volatile oil components extracted from Fritillaria cirrhosa D. Don was determined by area normalization. More than 39 components were separated and 14 components were identified from volatile oil in Fritillaria cirrhosa D. Don by comparing the obtained mass spectra of the analytes with NIST (National Institute of Standards and Technology)mass spectral database and standard spectra, among these compounds most of them were alkenes, alcohol and oxirane (Table-1).

Table-1 presents the summary results. The relative content of these constituents determined with area normalization method amount to $81.92 \%$ of the total amount of volatile oil. Comparison of the volatile oil compositions showed that the percentage of volatile chemical compounds from Fritillaria cirrhosa D. Don were different. The amounts of 1-octadecene and 1-dodecene which were significantly higher than other chemical compounds were up to $16.38 \%$ and $15.09 \%$, respectively.

Table-1 showed that the compounds determined concluded alkenes, alcohol and oxirane, etc., among which alkenes account for $31.47 \%$ of chromatographic peak area, alcohol compounds account for $20.61 \%$, oxirane compounds account for $11.38 \%$, ester compounds account for $11.35 \%$ acids account for $5.41 \%$. Compounds in which content was more than $5 \%$ concluded 1-octadecene (16.38\%), 1-dodecene (15.09), oxirane, hexadecyl-(11.38\%), 1-hexadecanol (10.65 $\%)$, 1-eicosanol (7.95\%), 9-octadecynoic acid, methyl ester (6.94\%), n-hexadecanoic acid (5.41\%). Other compounds identified in the sample extracts are listed in Table-1.

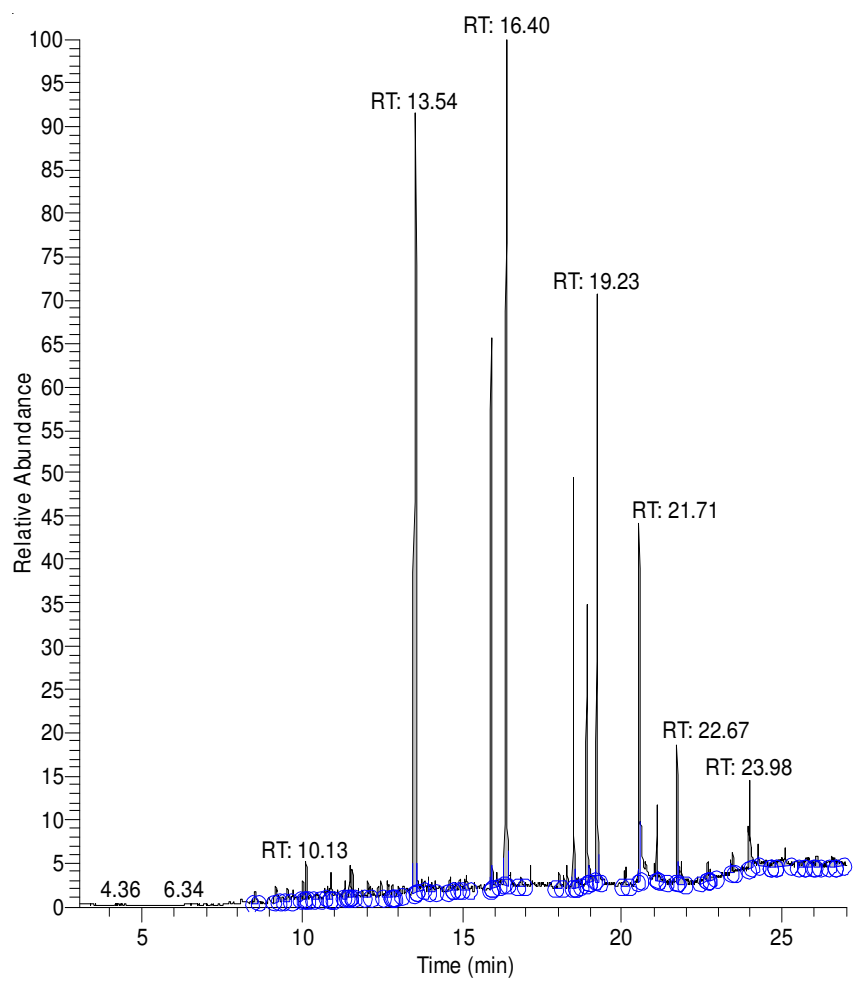

Fig. 2. Total ion current chromatograms of volatile oil of Fritillaria cirrhosa D.Don

TABLE-1

ANALYTICAL RESULT OF CHEMICAL CONSTITUENTS OF VOLATILE OIL OF Fritillaria cirrhosa D.Don

\begin{tabular}{cclc}
\hline Peak & $\begin{array}{c}\text { Residence } \\
\text { time (min) }\end{array}$ & \multicolumn{1}{c}{ Compound } & $\begin{array}{c}\text { Content } \\
(\%)\end{array}$ \\
\hline 1 & 10.13 & Benzofuran, 4,7-dimethyl- & 0.72 \\
2 & 11.48 & 3-Buten-2-one, 3-methyl-4-phenyl- & 0.62 \\
3 & 11.57 & 1-Phthalanol, 1,3,3-trimethyl- & 0.55 \\
4 & 13.54 & 1-Dodecene & 15.09 \\
5 & 15.90 & 1-Hexadecanol & 10.65 \\
6 & 16.40 & 1-Octadecene & 16.38 \\
7 & 18.49 & 1-Eicosanol & 7.95 \\
8 & 18.89 & n-Hexadecanoic acid & 5.41 \\
9 & 19.23 & Oxirane, hexadecyl- & 11.38 \\
10 & 20.53 & 9-Octadecynoic acid, methyl ester & 6.94 \\
11 & 21.08 & Ethanol, 2-(9-octadecenyloxy)-, (Z)- & 1.46 \\
12 & 21.71 & Z-2-Tetradecen-1-ol acetate & 2.68 \\
13 & 22.67 & Pentatriacontane & 0.36 \\
14 & 23.98 & bis-(2-Ethylhexyl) phthalate & 1.73 \\
\hline
\end{tabular}




\section{Conclusion}

In this paper, the volatile oil of Fritillaria cirrhosa D. Don has been investigated for the first time. By applying GCMS, the volatile oil of Fritillaria cirrhosa D.Don was analyzed and compared regarding their qualitative and relatively quantitative characteristics. The contents of volatile components extracted from Fritillaria cirrhosa D.Don are obviously different, which suggests that the identification of the volatile oil components is helpful to elucidate the active components and pharmacological activities. The information about the chemical constituents of volatile oils is useful and necessary to the further study of Fritillaria cirrhosa D.Don, which will certainly help us to utilize the traditional Chinese medicine.

\section{REFERENCES}

1. Pharmacopoeia Committee of Chinese Ministry of Health. Pharmacopoeia of Peoples Republic of China, People's Health Publishing House(Beijing) 25 (2005).

2. Y. Jiang, H.J. Li, P. Li, Z.H. Cai and W.C. Ye, J. Nat. Prod., 68, 264 (2005).

3. Y.H. Zhang, H.L. Ruan, H.F. Pi, J.Z. Wu, H.D. Sun and T. Fujita, J. Asian. Nat. Prod. Res., 6, 29 (2004).

4. S.L. Li, G. Lin, S.W. Chan and P. Li, J. Chromatogr. A, 909, 207 (2001).

5. G. Lin, P. Li, S.L. Li and S.W. Chan, J. Chromatogr. A, 935, 321 (2001).

6. H.J. Li, Y. Jiang and P. Li, Nat. Prod. Rep., 23, 735 (2006).

7. B.Q. Lin, H. Ji, P. Li, Y. Jiang and W. Fang, Eur. J. Pharmacol., 551, 125 (2006).

8. B.Q. Lin, H. Ji, P. Li and Y. Jiang, Planta Med., 72, 814 (2006).

9. K. Konchar, X.L. Li, Y.P. Yang and E. Emshwiller, Econ. Bot., 65, 283 (2011). 